\title{
Nonlinear behavior for nanoscale electrostatic actuators with Casimir force
}

\author{
Wen-Hui Lin ${ }^{\text {a }}$, Ya-Pu Zhao ${ }^{\text {b,* }}$ \\ a College of Science, China Agricultural University, Beijing 100083, PR China \\ ${ }^{\mathrm{b}}$ State Key Laboratory of Nonlinear Mechanics (LNM), Institute of Mechanics, Chinese Academy of Sciences, \\ Beijing 100080, PR China
}

Accepted 1 July 2004

Communicated by Prof. Ji-Huan $\mathrm{He}$

\begin{abstract}
The influence of Casimir force on the nonlinear behavior of nanoscale electrostatic actuators is studied in this paper. A one degree of freedom mass-spring model is adopted and the bifurcation properties of the actuators are obtained. With the change of the geometrical dimensions, the number of equilibrium point varies from zero to two. Stability analysis shows that one equilibrium point is Hopf point and the other is unstable saddle point when there are two equilibrium points. We also obtain the phase portraits, in which the periodic orbits exist around the Hopf point, and a homoclinic orbit passes through the unstable saddle point.
\end{abstract}

(c) 2004 Elsevier Ltd. All rights reserved.

\section{Introduction}

More than five decades ago, Hendrik Brugt Gerhard Casimir (1909-2000) predicts that the ground-state energy of photons is alternated in the presence of two parallel perfectly conducting metal plates in such a way as to lead to an observable macroscopic force between them [1]. His brief article discusses the discovery, formulation, physical significance and impact of one of the phenomena that bears his name, the eponymous Casimir effect. The regularized zero point energy per unit area between parallel plates of infinite conductivity separated by a distance $r$ is given by [1]:

$$
U(r)=\frac{\pi^{2} \hbar c}{720} \frac{1}{r^{3}},
$$

and the attractive Casimir force per plate area has universal amplitude as follows:

$$
\frac{F_{\mathrm{C}}}{A}=-\frac{\delta U}{\delta r}=\frac{\pi^{2} \hbar c}{240 r^{4}},
$$

\footnotetext{
* Corresponding author. Tel.: +86106265 8008; fax: +861062561284.

E-mail address: yzhao@Inm.imech.ac.cn (Y.-P. Zhao).
} 
where $\hbar$ is Planck's constant divided by $2 \pi$, and is equal to $1.055 \times 10^{-34} \mathrm{Js}, c$ is the speed of light and is equal to $2.998 \times 10^{8} \mathrm{~m} \mathrm{~s}^{-1}$. Because of its fundamental nature, the Casimir effect motivates extensive researches in the last decade. Potential applications of Casimir effect in nanoelectromechanical systems (NEMS) and nanotechnology are studied theoretically and experimentally [2-10]. It finds [5] that movable components in NEMS devices fabricated at distances less than $100 \mathrm{~nm}$ between each other often stick together due to strong Casimir force. Chan et al. [6,7] measure the Casimir force between a sphere and a flat plate in microelectromechanical systems (MEMS) using a micromachined torsional device, and the Casimir effect is demonstrated as a candidate for novel actuation source for the design of NEMS [6,7].

NEMS switches are fundamental building blocks for the design of NEMS applications, such as nanotweezers and some other nanoscale actuators [11,12]. There is an inherent instability, known as pull-in phenomenon, in both MEMS and NEMS switches. A typical MEMS switch is constructed from two conducting electrodes, one is typically fixed and the other is movable, which is suspended by using a mechanical spring. By applying a voltage difference between the two electrodes, the upper movable electrode displaces to the ground electrode because of the electrostatic force. At a certain voltage, the movable electrode becomes unstable and collapses (or pulls-in) to the ground plane. The voltage and gap distance of the switches at this state are called to as the pull-in voltage and the pull-in gap respectively, or together the pull-in parameters of switches.

An analytical expression of the pull-in parameters is given about the MEMS switches in [13]. A lumped two degrees of freedom pull-in model is presented in [14] for a direct calculation of the electrostatic actuators. The effect of residual charges, located in dielectric coating layers, upon the pull-in parameters of electrostatic actuators is studied in [15]. Axis stress, residual stress, and fringing-field effect have great influence on the behavior of RF switches, and even cause the failure of devices [16]. The pull-in phenomenon widely exists in many micromachined devices that require bi-stability for their operation, such as electrical RF switches [17] and some other micro-opto-electro-mechanical systems (MOEMS) devices [18]. The bifurcation analysis for an electrostatic microactuator is addressed in [19,20]. Dequesnes et al. study the pull-in voltage with van der Waals force in [21] while omitting its influence on the pull-in gap. In [22], Rokin considers the effect of van der Waals force on the pull-in gap, and gives the analytical expression of the pull-in gap and pull-in voltage with a general model. The dynamics behavior for nanoscale electrostatic actuators is studied by considering the effect of van der Waals force in [23]. The Casimir effect on the pull-in gap and pull-in voltage of NEMS switches is studied in [10]. An approximate analytical expression of the pull-in gap with the Casimir force is presented by the perturbation theory.

The present paper aims to study the bifurcation behavior of nanoscale electrostatic actuators considering the Casimir force and the maximum length of the switches that does not stick to the substrate is also determined. This study is believed to assist the understanding the actuation of NEMS switches by Casimir effect.

\section{The modeling system with Casimir force}

For simplicity and without loss of generality, the geometry shown in Fig. 1 is simplified to a one degree of freedom lumped model as shown in Fig. 2. The model consists of a linear spring, a mass, and a parallel-beam capacitor. Only one degree of freedom of the system is the gap distance, $r$, between the beam and the ground plane. This gap distance is defined to be the gap distance between the tip of cantilever structure and the ground plane for the cantilever switch and the gap distance between the center of the fixed-fixed structure and the ground plane for a fixed-fixed switch.

For the parallel beam configuration shown in Fig. 2, the electrostatic force (neglecting fringing fields) is given as

$$
F_{\text {elec }}=\frac{\varepsilon_{0} w L V^{2}}{2 r^{2}},
$$

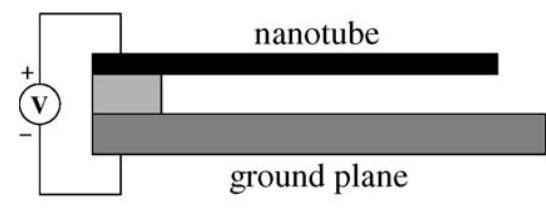

(a)

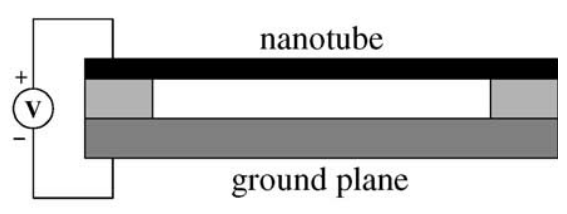

(b)

Fig. 1. Schematic of cantilever switch: (a) cantilever switch, and (b) fixed-fixed switch. 


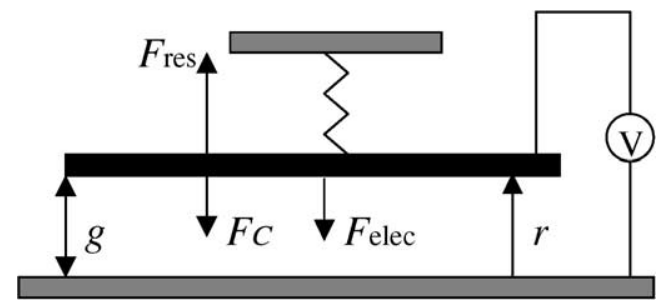

Fig. 2. One-dimensional lumped models for the pull-in parameters estimation.

where $\varepsilon_{0}$ is the permittivity of vacuum, $w$ is the width of the beam, $L$ is the length of the beam, and $V$ is the applied voltage. The restoring force of the beam is assumed to take the standard mass-spring form

$$
F_{\text {res }}=k(g-r) \text {, }
$$

where $k$ is the spring constant for the beam. It is defined as $k=F / r_{\max }$ in the continuum model, where $F$ is the uniform force applied on the beam and $r_{\max }$ is the maximum deflection of the beam. Thus, the spring constant depends on the cross-section shape as well as on the boundary conditions. Considering the model in this paper, the spring constant $k=8 E I / L^{3}$ for a cantilever structure, $k=384 E I / L^{3}$ for a fixed-fixed structure, $E$ is the Young's modulus, $I=w d^{3} / 12$ is the moment of inertia, and $d$ is the thickness of the beam. According to Eq. (2), the corresponding Casimir force of this model is

$$
F_{\mathrm{C}}=\frac{\pi^{2} \hbar c w L}{240 r^{4}} .
$$

The electrostatic force and Casimir force are attractive, the restoring force is repulsive. Their directions are shown in Fig. 2. According to Newton second law, we obtain the equation of motion of this model as

$$
m \frac{\mathrm{d}^{2} r}{\mathrm{~d} t^{2}}=F_{\text {res }}-F_{\text {elec }}-F_{\mathrm{C}}
$$

where $m$ is the mass of the beam. Introducing five dimensionless variables, $u=r / g, \tau=t / T, M=m / k T^{2}, a=\pi^{2} \hbar c w L / \mathrm{kg}^{5}$, $b=\varepsilon_{0} w L V^{2} / \mathrm{kg}^{3}$, and $T$ is the characteristic time, we transform the equation above into dimensionless form

$$
M \frac{\mathrm{d}^{2} u}{\mathrm{~d} \tau^{2}}=1-u-\frac{b}{2 u^{2}}-\frac{a}{240 u^{4}} .
$$

According to the definition of these parameters, physically meaningful solutions exist in the region $0<u<1$. The dimensionless parameter $a$ denotes the order of magnitude of ratio between the Casimir and the restoring forces, $b$ denotes the order of magnitude of ratio between the electrostatic and the restoring forces, $M$ denotes the order of magnitude of ratio between the inertia and the restoring forces.

\section{Pull-in parameters}

Setting zero the left-hand side of Eq. (7), one gets an equation as follows:

$$
f(u, a, b)=120 b u^{2}+a-240 u^{4}(1-u)=0 .
$$

The equilibrium is stable provided $\partial f(u) / \partial u<0$. As the voltage, i.e. the parameter $b$ increases, the gap distance decreases until instability or collapse condition is reached. By the critical condition $\partial f(u) / \partial u=0$ [24], one has

$$
\partial f(u) / \partial u=b u-4 u^{3}+5 u^{4}=0 .
$$

Combining Eqs. (8) and (9), we can solve the pull-in parameter $b_{\mathrm{PI}}=\varepsilon_{0} w L V_{\mathrm{PI}}^{2} / \mathrm{kg}^{3}$ which is related to the pull-in voltage $V_{\mathrm{PI}}$ and the pull-in gap $u_{\mathrm{PI}}$. Thus, we can discuss the pull-in parameter $b_{\mathrm{PI}}$ instead of the pull-in voltage $V_{\mathrm{PI}}$ for the given dimensions of this model. The pull-in parameter $b_{\mathrm{PI}}$ is given by

$$
b_{\mathrm{PI}}=\left[240 u_{\mathrm{PI}}^{4}\left(1-u_{\mathrm{PI}}\right)-a\right] \frac{1}{120 u_{\mathrm{PI}}^{2}},
$$


where $u_{\mathrm{PI}}$ should be computed by solving the following nonlinear equation:

$$
a+240 u_{\mathrm{PI}}^{4}-360 u_{\mathrm{PI}}^{5}=0 .
$$

If we neglect the contribution of the Casimir force in the analysis above, the pull-in gap and the pull-in parameter can be solved by Eqs. (10) and (11) as

$$
u_{0}=\frac{2}{3}, \quad \text { and } \quad b_{0}=\frac{8}{27},
$$

which are the same as the expressions derived in [13].

Numerical method can be used to solve the nonlinear equation (11). Perturbation theory was used in [10] to give an approximate analytical expression for the pull-in gap, that is

$$
\tilde{u}=u_{0}+\frac{9 a}{640} \text {. }
$$

The corresponding pull-in parameter is computed by

$$
\tilde{b}=\left[240 \tilde{u}^{4}(1-\tilde{u})-a\right] \frac{1}{120 \tilde{u}^{2}} .
$$

According to Eq. (11), we first plot the variation of the pull-in gap with parameter $a$ in Fig. 3. The variation of the corresponding pull-in parameter $b_{\mathrm{PI}}$ with parameter $a$ is drawn as Fig. 4 according to Eq. (10). In these two figures, there are two special points which are plotted by " $x$ " and "*", respectively. The point " $x$ " in Fig. 3 corresponds to $\left(a_{0}, u_{0}\right)=(0,2 / 3)$, when it is $\left(a_{0}, b_{0}\right)=(0,8 / 27)$ in Fig. 4 . This means that there is no effect of Casimir force on the actuators. With the appearance of the Casimir force, the pull-in gap $u_{\mathrm{PI}}$ increases when the pull-in parameter $b_{\mathrm{PI}}$ decreases. At another special point “*”, it corresponds to $\left(a^{*}, u^{*}\right)=(12,288 / 625,4 / 5)$, when it is $\left(a^{*}, b^{*}\right)=(12,288 / 625,0)$ in Fig. 4. That is, when $a>a^{*}, b$ will be negative. It means the actuator will lose its stability even though there is no voltage applied at the two electrodes.

In order to compare the linear approximation with nonlinear results, we also plot the linear approximate results in Figs. 3 and 4 according to Eqs. (13) and (14). From Figs. 3 and 4, we know the linear approximations are identical to nonlinear results when $27 a^{2} / 320 \ll 1$.

\section{The analysis of equilibrium points}

In Section 3, we discuss its critical condition in static state. In this section, we will discuss its dynamics behavior. First we set $y=\dot{u}$, Eq. (7) can be transformed into the following form:

$$
\frac{\mathrm{d} u}{\mathrm{~d} \tau}=y, \quad M \frac{\mathrm{d} y}{\mathrm{~d} \tau}=1-u-\frac{b}{2 u^{2}}-\frac{a}{240 u^{4}} .
$$

The equilibrium points are obtained by setting zero the left-hand sides of Eq. (15). The second equation is equivalent to Eq. (8), which has two parameters $a$ and $b$. Eq. (8) can be solved numerically for $u$ as a function of $a$ and $b$. First, we

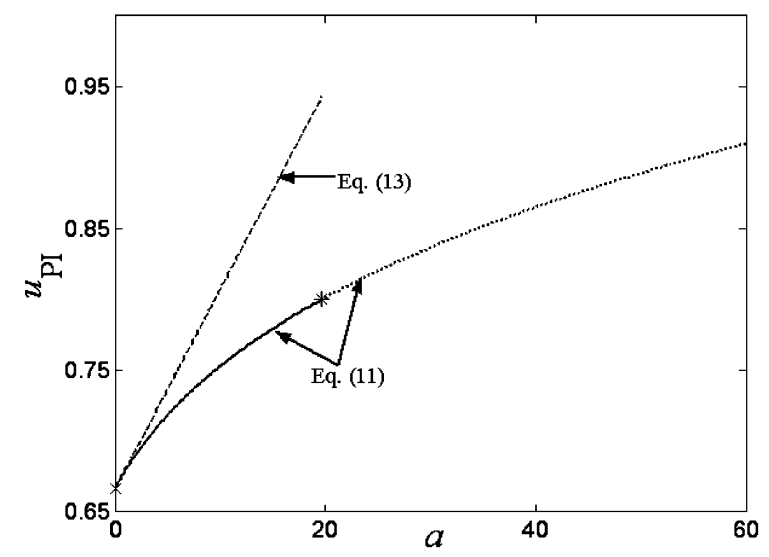

Fig. 3. Variation of the pull-in gap $u_{\mathrm{PI}}$ with parameter $a$. 


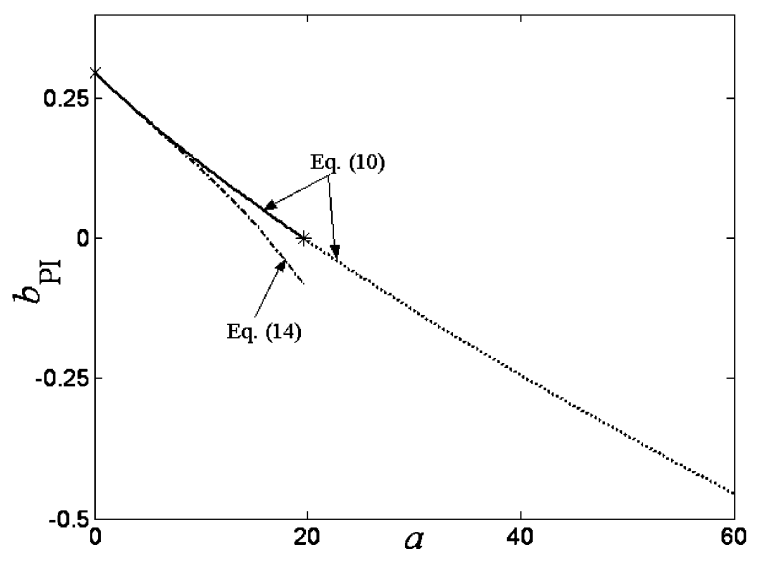

Fig. 4. Variation of the pull-in parameter $b_{\mathrm{PI}}$ with parameter $a$.

plot the variation of $u$ with parameter $b$ for the given different parameter $a$, the solution is shown in Fig. 5. From this figure, we know that: no solution exists in $0<u<1$ when $a$ is larger than the critical value $a^{*}$. When $a$ is less than $a^{*}$, two solutions exist in $0<u<1$ for any given $b \geqslant 0$. The critical value $a^{*}$ is determined in Section 3. From the discussion above, we know that Eq. (15) has two equilibrium points with parameter $a$ less than the critical value $a^{*}$ for any given $b$.

In order to check for their stability of the equilibrium points, the following Jacobian matrix is needed

$$
J=\left[\begin{array}{cc}
0 & 1 \\
\frac{b}{u^{3}}+\frac{a}{60 u^{5}}-1 & 0
\end{array}\right] .
$$

We first discuss the stability of the equilibrium points with the given parameters $b=0$ and $a<a^{*}$. According to Fig. 5, there are two equilibrium points $\left(u_{1}, 0\right)$ and $\left(u_{2}, 0\right)$ with satisfying this inequality $u_{1}>u^{*}>u_{2}$.

We first substitute $b=0, a<a^{*}$ and $u=u_{1}$ into the Jacobian matrix above, and get

$$
\left.J\right|_{u=u_{1}}=\left[\begin{array}{cc}
0 & 1 \\
\frac{a}{60 u_{1}^{5}}-1 & 0
\end{array}\right] .
$$

Its corresponding eigenvalue equation of $\left.J\right|_{u=u_{1}}$ satisfies $\lambda^{2}-\frac{a}{60 u_{1}^{5}}+1=0$. Due to $a<a^{*}$ and $u_{1}>u^{*}$, we know that $\lambda^{2}=\frac{a}{60 u_{1}^{5}}-1<0$. Then it has two pure imaginary roots, which means that the equilibrium point $\left(u_{1}, 0\right)$ is a Hopf point. Applying the same method to the other equilibrium point $\left(u_{2}, 0\right)$, its eigenvalue satisfies $\lambda^{2}=\frac{a}{60 u_{2}^{5}}-1>0$, then it has two real eigenvalues, one is positive, and the other is negative. It means this equilibrium point $\left(u_{2}, 0\right)$ is an unstable saddle point. Using the same method, we can discuss the stability of the two solutions with any different given $a$ and $b$. Then we

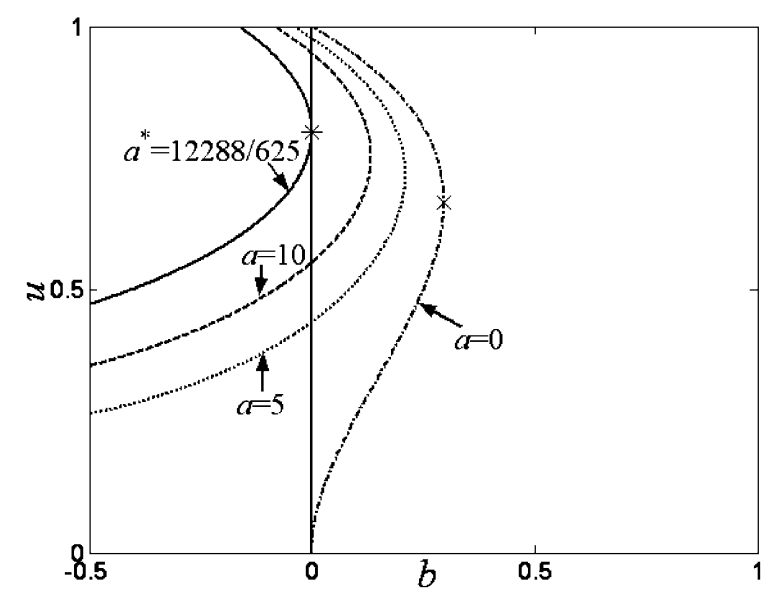

Fig. 5. Variation of equilibrium points with parameter $b$ for different $a$ values. 


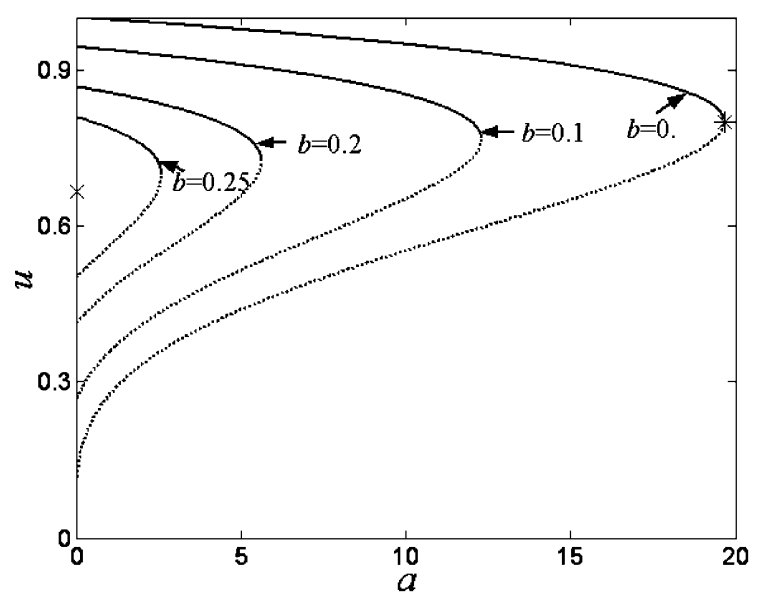

Fig. 6. Bifurcation diagram: variation of equilibrium points with parameter $a$ for given different $b$.

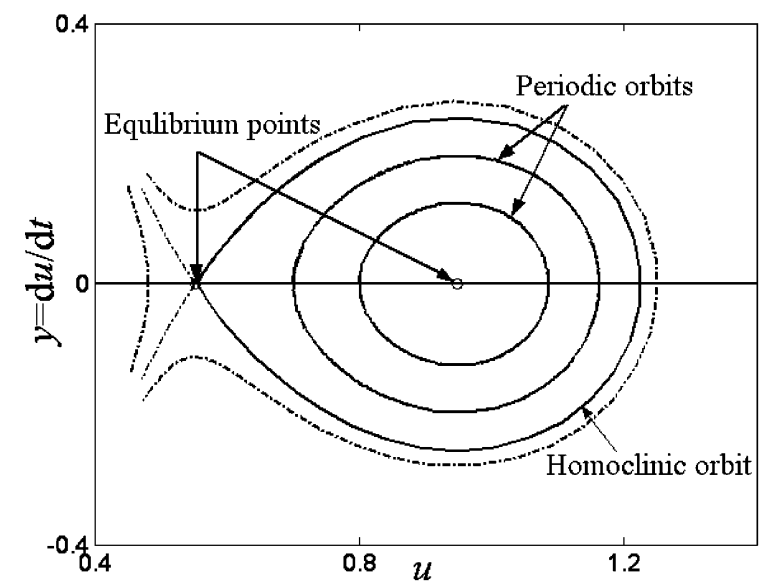

Fig. 7. Phase diagram with given $a=10$ and $b=0$.

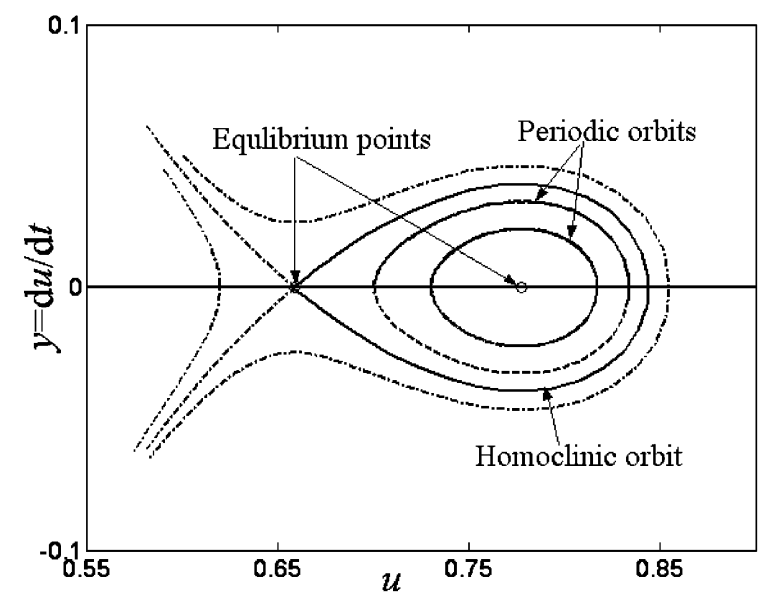

Fig. 8. Phase diagram with given $a=10$ and $b=1 / 5$. 


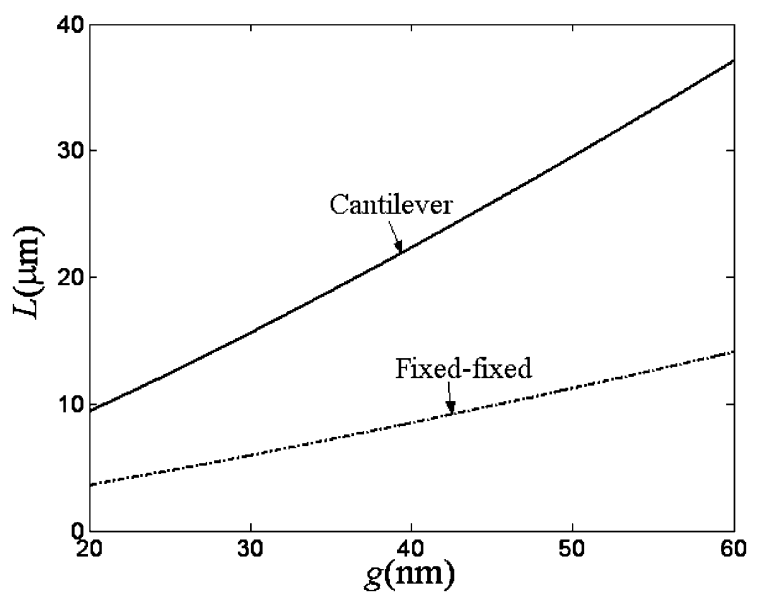

Fig. 9. Variation of the detachment length with the initial gap.

can plot its bifurcation diagram as Fig. 6. The real lines represent the Hopf point, and the dotted lines represent the unstable saddle point.

According to the property of the Hopf point and the unstable saddle point, we know that there exists periodic orbits around the Hopf point, and there is a homoclinic orbit passing through the unstable saddle point. Given different parameters $a$ and $b$, the corresponding phase portraits are plotted as Figs. 7 and 8, respectively.

\section{Detachment parameters}

The maximum length of the MEMS/NEMS structure without application of external voltage that will not stick to the substrate is called detachment length [23], which is obviously a basic design parameter for MEMS/NEMS. It is interesting to note that the detachment length of the cantilever and fixed-fixed beam can be obtained by critical value $a^{*}=12,288 / 625$. That is, the detachment length of the cantilever beam that will not adhere with the substrate due to Casimir force is

$$
L_{\max }=\frac{8 g}{5} \sqrt[4]{\frac{2 E d^{3} g}{\pi^{2} \hbar c}}
$$

and the detachment length of the fixed-fixed beam is

$$
L_{\max }^{\prime}=\frac{16 g}{5} \sqrt[4]{\frac{6 E d^{3} g}{\pi^{2} \hbar c}} .
$$

From Fig. 9, one can see the variation of the maximum length with the initial gap distance of the cantilever and fixed-fixed switches, respectively. As an alternative case, if the length is known, we can calculate the minimum gap, $g_{\min }$, between the switch and the substrate to ensure that the switch does not adhere to the substrate due to the Casimir force.

\section{Conclusions}

A theoretical analysis is presented for the influence of Casimir effect on the nonlinear behavior of nanoscale electrostatic actuators.

We first study the variation of pull-in parameters $u_{\mathrm{PI}}$ and $b_{\mathrm{PI}}$ with parameter $a$. From Figs. 3 and 4 , we know that the movable beam will collapse onto the ground plane without any voltage applied at the two electrodes when $a \geqslant a^{*}$. From the expression of critical value $a^{*}$, we can obtain the detachment length $L_{\max }=8 g \sqrt[4]{2 E d^{3} g / \pi^{2} \hbar c} / 5$ for cantilever type switch, and $L_{\max }^{\prime}=16 g \sqrt[4]{6 E d^{3} g / \pi^{2} \hbar c} / 5$, for fixed-fixed type switch. The minimum gap between the switch and the substrate of the nanoscale actuators can be derived in the same manner for the given switch length. 
We then study the equilibrium points and their corresponding stability of Eq. (15) when considering $a$ and $b$ as two parameters. From Fig. 5, no solution exists in $0<u<1$ satisfying $b \geqslant 0$ when $a \geqslant a^{*}$. There are two equilibrium points for any $b \geqslant 0$ when $a<a^{*}$. By discussing the stability of its equilibrium points, it is noted that one equilibrium point is a Hopf point, and the other is an unstable saddle point. Thus Eq. (15) has periodic orbits around the Hopf point, and a homoclinic orbit passing though the unstable saddle point seen by Figs. 7 and 8 .

By using a simple mass-spring model we show that there exists bifurcation as the geometry of the device is changed. Compared these results with [18,19], Casimir force will not change the number of equilibrium points and their corresponding stability. The attractive nature of the Casimir force just decreases the pull-in voltage.

\section{Acknowledgments}

The authors acknowledge the financial support of Chinese Academy of Sciences (No. KJCX2-SW-L2) and the National Natural Science Foundation of China (Grant Nos. 10225209, 50131160739 and 10072068).

\section{Appendix A}

Main parameters in the present paper

\begin{tabular}{lll}
\hline Symbol & Physical meaning & Dimension \\
\hline$a$ & $\pi^{2} \hbar c w L / \mathrm{kg}^{5}$, order of magnitude of ratio of Casimir and restoring forces & Dimensionless \\
$b$ & $\varepsilon_{0} w L V^{2} / \mathrm{kg}^{3}$, order of magnitude of ratio of electrostatic and restoring forces & Dimensionless \\
$c$ & Speed of light & $L T^{-1}$ \\
$d$ & Thickness of beam & $L$ \\
$E$ & Effective modulus of beam & $M L^{-1} T^{-2}$ \\
$g$ & Initial gap between movable and ground plates & $L$ \\
$\hbar$ & Planck's constant divided by $2 \pi$ & $M L^{2} T^{-1}$ \\
$I$ & Moment of the inertia of cross-section & $L^{4}$ \\
$k$ & Effective spring constant of beam $k=8 E I / L^{3}$ for cantilever & $M T^{-2}$ \\
& $k=384 E I / L^{3}$ for fixed-fixed beam & \\
$L$ & Length of beam & $L$ \\
$L_{\text {max }}$ & Detachment length of actuator & $L$ \\
$M$ & $m / k T^{2}$ & Dimensionless \\
$r$ & Gap between movable and ground plates & $L$ \\
$T$ & Characteristic time & $T$ \\
$u$ & $r / g$ & Dimensionless \\
$w$ & Width of beam & $L$ \\
$V$ & Voltage applied & $M L^{2} T^{-2} Q^{-1}$ \\
$\varepsilon_{0}$ & Vacuum permittivity & $\mathrm{M}^{-1} L^{-3} T^{2} Q^{2}$ \\
$\tau$ & $t / T$ & Dimensionless \\
\hline
\end{tabular}

\section{References}

[1] Casimir HBG. On the attraction between two perfectly conducting plates. Proc K Ned Akad Wet 1948;51:793-6.

[2] Bordag M, Mohideen U, Mostepanenko VM. New developments in the Casimir effect. Phys Rep 2001;353:1-205.

[3] Kenneth O et al. Repulsive Casimir force. Phys Rev Lett 2002;89:033001.

[4] Serry FM, Walliser D, Maclay GJ. The role of the Casimir effect in the static deflection and stiction of membrane strips in MEMS. J Appl Phys 1998;84:2501-6.

[5] Bukes E, Roukes ML. Stiction, adhesion energy, and the Casimir effect in micromechanical systems. Phys Rev B 2001;63:033402.

[6] Chan HB et al. Quantum mechanical actuation of microelectromechanical systems by the Casimir force. Science 2001;291: $1941-4$.

[7] Chan HB et al. Nonlinear micromechanical Casimir oscillator. Phys Rev Lett 2001;87:211801. 
[8] Zhao YP, Wang LS, Yu TX. Mechanics of adhesion in MEMS—a review. J Adhes Sci Technol 2003;17:519-46.

[9] Zhao YP, Li WJ. Surface stability of epitaxial elastic films by the Casimir force. Chin Phys Lett 2002;19:1161-3.

[10] Lin WH, Zhao YP. Casimir effect on the pull-in parameters of nanometer switches. Microsyst Technol, in press.

[11] Kim P, Lieber CM. Nanotube nanotweezers. Science 1999;286:2148-50.

[12] Akita $\mathrm{S}$ et al. Nanotweezers consisting of carbon nanotubes operating in an atomic force microscope. Appl Phys Lett 2001;79:1691-3.

[13] Osterberg PM. Electrostatically actuated micromechanical test structures for material property measurement. PhD Dissertation MIT, Cambridge, MA, 1995.

[14] Bochobza-Degani O, Nemirovsky Y. Modeling the pull-in parameters of electrostatic actuators with a novel lumped two degrees of freedom pull-in model. Sensors Actuat A 2002;97-98:569-78.

[15] Bochobza-Degani O, Socher E, Nemirovsky Y. On the effect of residual charges on the pull-in parameters of electrostatic actuators. Sensors Actuat A 2002;97-98:563-8.

[16] Zhang LX, Zhang JW, Zhao YP, Yu TX. Failure modes of doubly supported capacitive RF MEMS switches. Int J Nonlinear Sci Numer Simul 2002;3(3/4):353-6.

[17] Zhang LX, Zhao YP. Electromechanical model of RF MEMS switches. Microsyst Technol 2003;9:420-6.

[18] Hornbeck LJ. Spatial light modulator and method. US Patent 5,061,049, 1991.

[19] Pelesko JA. Multiple solutions in electrostatic MEMS. In: Proc Modeling Simulation Microsystems (MSM), 2001. p. $290-3$.

[20] Bernstein D, Guidotti P, Pelesko JA. Mathematical analysis of an electrostatically actuated MEMS device. In: Proc. Modeling Simulation Microsystems (MSM), 2000. p. 489-92.

[21] Dequesnes M, Rotkin SV, Aluru NR. Calculation of pull-in voltages for carbon-nanotube-based nanoelectromechanical switches. Nanotechnology 2002;13:120-31.

[22] Rotkin SV. Analytical calculations for nanoscales electromechanical systems. Electrochem Soc Proc 2002;6:90-7.

[23] Lin WH, Zhao YP. Dynamics behavior of nanoscale electrostatic actuators. Chin Phys Lett 2003;20:2070-3.

[24] Seydel R. Practical bifurcation and stability analysis: from equilibrium to chaos. 2nd ed. Interdisciplinary applied mathematics, vol. 5. Springer-Verlag; 1994. 